\title{
Innere Medizin und Qualitätsförderung
}

\author{
M. Stäubli
}

«Schon eine Woche vor dem Absturz der ¿Columbia` bezweifelten Nasa-Ingenieure die Flugtüchtigkeit des Space Shuttles. Präzise sagten einige von ihnen den Hergang des Unfalls vom 1. Februar 2003 voraus. Doch sie informierten weder ihre Vorgesetzten noch die todgeweihte Besatzung.» [1]

Dieses Zitat erhellt schlaglichtartig und exemplarisch, was passieren kann, wenn frühe Warnzeichen einer möglichen Katastrophe nicht beachtet bzw. nicht ernstgenommen werden und wenn die Kommunikation zwischen Spezialistengruppen nicht funktioniert, mit anderen Worten, wenn die Schnittstellen nicht eindeutig definiert und gesichert sind. Man spricht in solchen Fällen oft von einer Verkettung unglücklicher Umstände. Im nachhinein wird klar, dass man es versäumt hat, die glücklichen und systemerhaltenden Umstände sicher und rechtzeitig miteinander zu verketten.

Die Innere Medizin ist ein Fach mit vielen Schnittstellen. Sie ist es zwangsläufig aufgrund ihres organübergreifenden Charakters, durch den sie im Kern definiert ist. Je ausgeprägter die Polymorbidität der Patientinnen und Patienten, desto notwendiger ist die Innere Medizin, welche die Schnittstellen sichert, oder anders ausgedrückt, Risse und Bruchstellen im Umfeld einer organspezialisierten interdisziplinären $\mathrm{Pa}$ tientenbetreuung verhindert.

Mit dem Problem der Schnittstellensicherung ist das Problem der Patientensicherheit auf das engste verbunden. Schnittstellen stellen für Patientinnen und Patienten in der Tat die gefährlichsten Bereiche innerhalb von Teamarbeit und Prozessen dar. Die immer kürzeren Arbeitszeiten und die Spezialisierung auch im paramedizinischen Bereich führen zur Zunahme der Anzahl Möglichkeiten von Übermittlungspannen. Das Problem ist nicht spezifisch für die Innere Medizin, doch kumuliert es hier, weil die Polymorbidität der internistischen Patientinnen und $\mathrm{Pa}$ tienten viele Organspezialisten auf den Plan ruft. Der Schnittstellenproblematik muss deshalb besondere Aufmerksamkeit geschenkt werden. Dazu gehört vor allem auch die Zusammenarbeit mit technisch interventionellen Fächern, wie zum Beispiel der Gastroenterologie und der interventionellen Radiologie. Hier dürfen die Internisten z.B. die Patientenaufklärung über geplante invasive Prozeduren nicht den Spezialisten allein überlassen, sondern sie müssen wissen, was sie ihren Patienten zumuten, d.h. die Risiken kennen und deshalb fähig sein, eine entsprechende Aufklärung durchzuführen, beispielsweise zur perkutanen Biopsie viszeraler Organe oder zu endoskopischen Verfahren.

Mit der Pflege der Schnittstellen ist auch die Beziehung zu den Patienten gemeint: es ist ausserordentlich wichtig, dass zum Beispiel die Information der Patientinnen und Patienten über die Wirkungen und Nebenwirkungen der verordneten Medikamente von ärztlicher Seite gut und umfassend durchgeführt wird. Tun wir dies nicht, werden sich neben den Pharmazeuten noch weitere Bereiche wie etwa der Pflegebereich um diese Dinge kümmern wollen und den Internisten dieses wichtige Gebiet eines Tages streitig zu machen versuchen. Unsere Hauptwaffe sind die Medikamente. Der Pharmakotherapie muss unsere ganz besondere und verstärkte Aufmerksamkeit gelten.

Es sollte zu einer Selbstverständlichkeit werden, dass die internistische Arbeit berufs- und alltagsbegleitend in bezug auf ihre Qualität evaluiert wird: dies im Sinne einer permanenten fachlichen Qualitätskontrolle. In einer Weiterbildungsstätte muss z.B. ein entgleister Quickwert bezüglich der Ursache der Entgleisung stets hinterfragt werden, um ähnliche Entgleisungsmechanismen für andere Fälle vorauszusetzen und Entgleisungen zu verhindern. Es ist notwendig, dass die Internisten solche Programme selber durchführen; wir dürfen nicht warten, bis behördlicherseits auch noch fachliche Qualitätskontrollen vorgeschrieben werden; es ist schon genug, wenn sich die nicht-medizinische Bürokratie in die Qualitätskontrolle des medizinischen Patientenmanagements in den Spitälern einschaltet.

Qualitätsförderung in der Inneren Medizin ist nicht durch behördliche Obligatorien, Reglemente und Broschüren möglich, sondern sie basiert auf der eigenverantwortlichen Arbeit der Fachleute in den Praxen, Spitälern, Universitäten und den fachärztlichen Gesellschaften.

In diesem Sinne betreibt die Schweizerische Gesellschaft für Innere Medizin (SGIM) das Programm «Patientenzufriedenheit» mit dem Verein ARPAZ, eine laufende Evaluation der ambulanten Praxisqualität durch die Patienten; die Chefärztevereinigung registriert und evaluiert mit dem interkantonalen Programm «Komplikationenliste» die Komplikationen medizinischer Interventio- 
nen in den Spitälern zwecks Verbesserung der Patientensicherheit; der Vorstand der SGIM hat eine Charta zur Verbesserung der Zusammenarbeit zwischen Praxis und Spital ausgearbeitet.

Eine der grossen Herausforderungen an die Innere Medizin in den nächsten zwei Jahrzehnten ist die Polymorbidität der immer älter werdenden Bevölkerung. Laut Statistik des Bundesamtes für Statistik, BFS, nimmt die Zahl der über 65jährigen Leute von 15\% im Jahr 2000 auf 20\% im Jahr 2020 und auf $23 \%$ im Jahr 2030 zu. Diese Entwicklung bedingt die Anpassung von Strukturen - selbstverständlich nicht nur im Betreuungsbereich der Inneren Medizin - und des Know-hows im Gebiet der internistischen Pharmakotherapie. Als internistisches Beispiel von zunehmender Brisanz für die Zukunft sei hier vor allem die Herzinsuffizienz mit all ihren assoziierten Problemen und Komplikationen genannt.

Das gemeinsame Programm der diesjährigen Jahresversammlung der Schweizerischen Gesellschaft für Innere Medizin und verwandter Fachgesellschaften im Mai 2003 in Basel nimmt Bezug auf verschiedene Aspekte der angesprochenen Problematik. So wird am standespolitischen Seminar über die Entwicklung der Inneren Medizin im Zusammenhang mit den Veränderungen an den internistischen Universitätskliniken debattiert. Die Sorge gilt dabei den drohenden Aufteilungen internistischer Departemente in andere Spezialdisziplinen, was die umfassende internistische Weiterbildung an den Universitäten in Frage stellt.
Im wissenschaftlichen Programm wird auf die wichtige pharmakotherapeutische Seite internistischer Tätigkeit eingegangen, so insbesondere mit dem interessanten Seminar von S. Krähenbühl zu klinisch relevanten Arzneimittelinteraktionen. Dieser Beitrag vermittelt unter anderem auch, dass die immer beängstigendere Fülle neuer Erkenntnisse fast ultimativ nach neuen Hilfsmitteln für die tägliche Pharmakotherapie verlangt, vor allem für die polypharmakologische Behandlung multimorbider internistischer Patienten. Solche Informationen steuert der Workshop zur «Medikamentenverschreibung im Internetzeitalter» von M. Kondo Oestreicher bei.

Mit den Fragen von C. Chappuis: «Rationierte Medizin bei Hochbetagten?», H. B. Stähelin: «Lässt sich das Altern verzögern?» und A. Stuck: «Wie können wir die Selbständigkeit im Alter erhalten?» beleuchten diese Referenten das Spannungsfeld der Qualität des Lebens und der Quantität an Jahren, aber auch der Quantität an Mitteln zur Ermöglichung dessen, was viele möchten: Alt werden, aber nicht alt sein, und wenn schon, dann gesund. Also: Jede Altersstufe ist von diesen Themen zur zukünftigen Lebensqualität angesprochen. Die Qualität der Inneren Medizin der Zukunft wird unter anderem daran zu messen sein, wie sie ihre Rolle im Zusammenhang mit den multiplen Problemen der älteren Bevölkerung erfüllen wird. Die Aussicht, dass weniger Geld und womöglich weniger Technik zur Verfügung sein werden, darf aber nicht heissen, dass es auch weniger an Zeit und an Menschlichkeit sein soll.
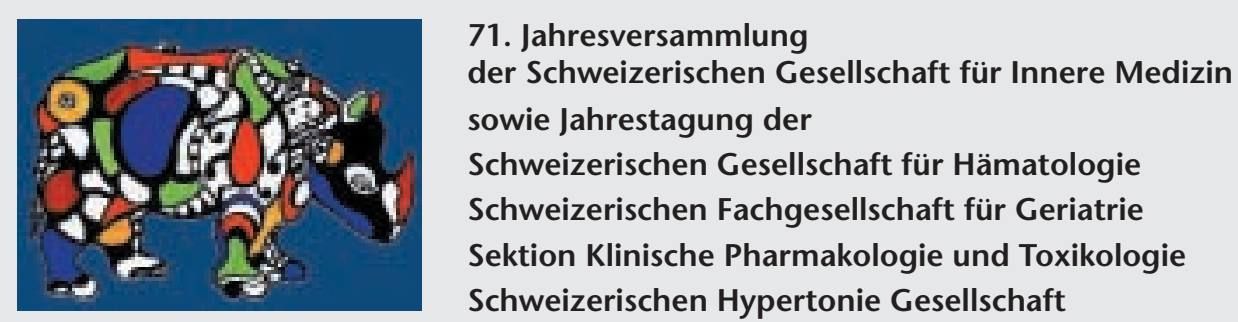

Ort und Datum: Basel, Mittwoch, 21. Mai, bis Freitag, 23. Mai 2003

Der Klinische Track soll Gelegenheit geben, nicht nur das Neueste, sondern das Entscheidendste zu erfahren.

Clinical year in review: Exponenten aus verschiedenen Fachgebieten der Medizin bieten einen Überblick über neueste wissenschaftliche und klinisch relevante Erkenntnisse ihres Faches.

Clinical pearls: Chefärzte der Nordwestschweiz präsentieren ausgewählte Fälle.

Workshops: Grundversorger und Experten bearbeiten gemeinsam mit dem Publikum ein Thema in kleinen Gruppen.

Skill lab: Informatik, Blutdruckmessung, Gastroskopie, Bronchoskopie, Inhalationstechniken, Prostatapalpation, Selbststudiumprogramme in Radiologie, Ergonomie am Arbeitsplatz.

Sachverstandprüfung im Strahlenschutz BAG: 21. Mai, 11.45 Uhr.

Administratives Sekretariat: Jahresversammlung SGIM 2003, c/o AKM Congress Service, Postfach, 4005 Basel, Tel. 06168677 11, Fax 06168677 88, E-Mail: info@akm.ch

Website mit weiteren Infos: www.akm.ch/sgim2003 